\title{
STATIONARY QUANTUM MARKOV PROCESSES AS SOLUTIONS OF STOCHASTIC DIFFERENTIAL EQUATIONS
}

\author{
JÜRGEN HELLMICH \\ Mathematisches Institut, Universität Tübingen \\ Auf der Morgenstelle 10, D-72 076 Tübingen, Germany \\ E-mail: Juergen.Hellmich@uni-tuebingen.de \\ CLAUS KÖSTLER \\ Mathematisches Institut, Universität Tübingen \\ Auf der Morgenstelle 10, D-72 076 Tübingen, Germany \\ E-mail: koestler@mathematik.uni-stuttgart.de \\ BURKHARD KÜMMERER \\ Mathematisches Institut A, Universität Stuttgart \\ Pfaffenwaldring 57, D-70 569 Stuttgart, Germany \\ E-mail:kuem@mathematik.uni-stuttgart.de
}

\begin{abstract}
From the operator algebraic approach to stationary (quantum) Markov processes there has emerged an axiomatic definition of quantum white noise. The role of Brownian motion is played by an additive cocycle with respect to its time evolution. In this report we describe some recent work, showing that this general structure already allows a rich theory of stochastic integration and stochastic differential equations. In particular, if a quantum Markov process is represented by a unitary cocycle, we can reconstruct an additive cocycle ('quantum Brownian motion') and the unitary cocycle ('quantum Markov process') appears as the solution of a certain stochastic differential equation. This establishes a one-to-one correspondence between multiplicative and additive adapted cocycles. As an application of this result we construct stationary Markov processes, driven by squeezed white noise and $q$-white noise.
\end{abstract}

1991 Mathematics Subject Classification: Primary 46L50; Secondary 81S25, 60H07.

This paper is an extended version of a talk given at the Quantum Probability Conference in Gdańsk (July 1997) by the second author.

This work is part of a research project supported by the Deutsche Forschungsgemeinschaft. Research of the second author supported by Ev. Studienwerk Villigst e.V.

The paper is in final form and no version of it will be published elsewhere. 
1. Introduction. In the theory of stochastic processes Brownian motion frequently appears as the driving force for more general Markov processes. This idea is made rigorous within the framework of stochastic calculus. It allows one to construct Markov processes as solutions of stochastic differential equations.

The needs to cover also stochastic phenomena governed by the rules of quantum theory led to the development of quantum probability, thus extending classical probability. An early success of this theory was an extension of the Itô stochastic integral with respect to Brownian motion by R. Hudson and K.R. Parthasarathy. It allows also Bosonic or Fermionic creation, annihilation and gauge processes (in the Bosonic case) as increment process. For an account of this theory we refer to $[\mathrm{P}, \mathrm{Me}]$. Meanwhile, quantum stochastic calculus has been further developed. Of particular importance for the present approach are $[\mathrm{AFQ}, \mathrm{ApFr}, \mathrm{BiSp}, \mathrm{BSW}, \mathrm{HuLi}, \mathrm{KüSp}]$. The starting point for the present paper was $[\mathrm{Pr}]$.

As in the classical theory, quantum stochastic calculus could be used for constructing certain quantum Markov processes as solutions of quantum stochastic differential equations. To be more precise, solving such a stochastic differential equation yields a family $\left(u_{t}\right)_{t \geq 0}$ of unitaries on a Hilbert space $\mathcal{H}_{0} \otimes \mathcal{F}$ with $\mathcal{F}$ a Bosonic or Fermionic Fock space (meanwhile also $q$-deformed Fock spaces have been considered in [BoSp, BKS]). The unitaries satisfy a cocycle identity with respect to the shift evolution on $\mathcal{F}$, the 'evolution of white noise'. With the 'initial algebra' $\mathcal{A}_{0}:=\mathcal{B}\left(\mathcal{H}_{0}\right)$ the family $\left(j_{t}\right)_{t \geq 0}$ of 'noncommutative random variables' given by $j_{t}: \mathcal{B}\left(\mathcal{H}_{0}\right) \ni x \mapsto j_{t}(x):=u_{t}^{*}(x \otimes 1) u_{t}$ is a quantum Markov process in the sense of $[\mathrm{AFL}]$.

On the other hand, there has been an operator algebraic approach to the theory of stationary quantum Markov processes ([Kü1, Kü2]). Here, it could be shown in great generality, that from a quantum Markov process there can be extracted a noise algebra equipped with a stationary evolution. Its properties are encoded into the axiomatic definition of white noise (cf. Section 3). Moreover, the Markovian time evolution appears as a perturbation of the white noise evolution by an adapted unitary cocycle. An example of such a result from [Kü2] is mentioned in Theorem 3.2. The class of such processes is much wider than the ones on Fock spaces mentioned above. So the question arises, whether even in this great generality, these processes can still be identified with solutions of certain stochastic differential equations. In the following we answer this question in the affirmative.

The answer has to have two parts: From a quantum Markov process, i.e. its unitary cocycle, one first has to reconstruct an increment process, playing the role of Brownian motion. It appears in the guise of an additive cocycle with respect to the white noise evolution. Secondly, one has to show that for any additive cocycle one can define stochastic Itô type integrals leading to the familiar results on stochastic differential equations. From such a stochastic differential equation one should retain the unitary cocycle as its solution. In combining these parts we end up with a one-to-one correspondence between unitary cocycles and additive cocycles as stated in our main Theorem 6.1.

It turns out that for the present context it is appropriate not to separate the initial algebra from the noise algebra. Instead, it is convenient to describe the theory in the language of Hilbert modules. 
Let us finally summarise the content of this paper.

First of all, one has to look for adequate definitions of stochastic independence and quantum white noise in the general setting. This is the topic of Section 3 after fixing some basic notions in Section 2.

Second, a theory of stochastic integration has to be established. For this purpose we introduce a Hilbert module of quantum white noise. This already allows us to give a bijective correspondence between additive and isometric Hilbert module cocycles (Section 4).

In a third step we give in Section 5 a noncommutative generalization of the classical Itô correction $\left(d B_{t}\right)^{2}=d t$ of Brownian motion.

In Section 6 we present the main result, a bijective correspondence between additive and unitary cocycles with respect to the free evolution of a quantum white noise. This immediately leads to the construction of stationary quantum Markov processes.

Finally, in Section 7 we apply the general theory to the examples of squeezed white noise and $q$-white noise.

2. Basic notions of quantum probability. We introduce some fundamental notions and conventions which will be used throughout the following. For a more explicit description of the concepts of quantum probability we refer to [AFL, Kü1]; for an elementary introduction cf. [KüMa].

A (noncommutative or quantum) probability space is given by a pair $(\mathcal{A}, \varphi)$ with $\mathcal{A}$ a von Neumann algebra and $\varphi$ a faithful normal state on $\mathcal{A}$. The natural morphisms $T:(\mathcal{A}, \varphi) \rightarrow(\mathcal{B}, \psi)$ between two probability spaces are given by completely positive operators $T: \mathcal{A} \rightarrow \mathcal{B}$ such that $T\left(\mathbb{1}_{\mathcal{A}}\right)=\mathbb{1}_{\mathcal{B}}$ and $\psi \circ T=\varphi$. By $\operatorname{Aut}(\mathcal{A}, \varphi)$ we denote the automorphisms of $(\mathcal{A}, \varphi)$. We understand a conditional expectation $P$ from $(\mathcal{A}, \varphi)$ onto some von Neumann subalgebra $\mathcal{B}$ of $\mathcal{A}$ as an endomorphism of $(\mathcal{A}, \varphi)$. The conditional expectation $P:(\mathcal{A}, \varphi) \rightarrow \mathcal{B}$ exists (and is unique) iff $\mathcal{B}$ is globally invariant under the modular automorphism group $\sigma_{t}^{\varphi}$. We denote the centralizer of $(\mathcal{A}, \varphi)$ by $\mathcal{A}^{\varphi}$ and the commutant of $\mathcal{A}$ by $\mathcal{A}^{\prime}$. If $\mathcal{A}_{1}$ and $\mathcal{A}_{2}$ are von Neumann subalgebras of $\mathcal{A}$ then $\mathcal{A}_{1} \bigvee \mathcal{A}_{2}$ denotes the von Neumann subalgebra of $\mathcal{A}$ which is generated by $\mathcal{A}_{1}$ and $\mathcal{A}_{2}$. Similarly, $\bigvee_{i \in I} \mathcal{A}_{i}$ is defined for a family $\left(\mathcal{A}_{i}\right)_{i \in I}$ of subalgebras.

If for any interval $I \subset \mathbb{R}, \mathcal{A}_{I}$ is a von Neumann subalgebra of $\mathcal{A}$, such that (i) the conditional expectation $P_{I}$ from $(\mathcal{A}, \varphi)$ onto $\mathcal{A}_{I}$ exists, (ii) $I_{1} \subseteq I_{2}$ implies $\mathcal{A}_{I_{1}} \subseteq \mathcal{A}_{I_{2}}$, and finally (iii) $\mathcal{A}_{[r, t]}=\mathcal{A}_{[r, s]} \bigvee \mathcal{A}_{[s, t]}$ whenever $r \leq s \leq t$, then we call $\left(\mathcal{A}_{I}\right)_{I}$ a filtration of the probability space $(\mathcal{A}, \varphi)$. A filtration $\left(\mathcal{A}_{I}\right)_{I}$ of the probability space $(\mathcal{A}, \varphi)$ is called an $\mathcal{A}_{0}$-Markov filtration, if $P_{(-\infty, 0]}{ }^{\circ} P_{[0, \infty)}=P_{0}\left(P_{0}\right.$ denotes the conditional expectation from $(\mathcal{A}, \varphi)$ onto $\left.\mathcal{A}_{0}\right)$. We remark that $\mathcal{A}_{0}$ can be viewed as the image of an identity preserving injective *-homomorphism $i:(\mathcal{B}, \psi) \rightarrow(\mathcal{A}, \varphi)$ which is called a noncommutative random variable. Here we always identify $\mathcal{B}$ with its embedding $\mathcal{A}_{0}$ in $\mathcal{A}$.

A stationary dynamical system $\left(\mathcal{A}, \varphi, T_{t}\right)$ is given by a probability space $(\mathcal{A}, \varphi)$ and a pointwise weak*-continuous group $\left(T_{t}\right)_{t \in \mathbb{R}} \subset \operatorname{Aut}(\mathcal{A}, \varphi)$. A stationary (quantum) Markov process $\left(\mathcal{A}, \varphi, T_{t} ; \mathcal{A}_{0}\right)$ with values in $\mathcal{A}_{0}$ is a stationary dynamical system $\left(\mathcal{A}, \varphi, T_{t}\right)$ such that there exists an $\mathcal{A}_{0}$-Markov filtration $\left(\mathcal{A}_{I}\right)_{I}$ and $\bigvee_{s \in[-r, t]} T_{s}\left(\mathcal{A}_{0}\right) \subseteq \mathcal{A}_{[-r, t]}$ for any $-r \leq 0 \leq t$. Defining $R_{t}:=P_{0} \circ T_{t} \circ P_{0}$, every stationary Markov process $\left(\mathcal{A}, \varphi, T_{t} ; \mathcal{A}_{0}\right)$ with values in $\mathcal{A}_{0}$ yields an irreversible stationary dynamical system $\left(\mathcal{A}_{0}, \varphi, R_{t}\right)$. 
Finally we agree upon the following general conventions. If $(\mathcal{A}, \varphi)$ is a probability space we denote its GNS Hilbert space by $L^{2}(\mathcal{A}, \varphi)$ and the state $\varphi$ is represented as a vector state by the cyclic separating vector $\Omega \in L^{2}(\mathcal{A}, \varphi)$, i.e. $\varphi=\langle\Omega \mid \cdot \Omega\rangle$. If $u \in \mathcal{A}$ is any unitary then $\operatorname{Ad} u$ denotes the inner automorphism $\mathcal{A} \ni x \mapsto u^{*} x u$ of $\mathcal{A}$. The complex $n \times n$-matrices are denoted by $M_{n}$ where $n=\infty$ is allowed.

3. Stochastic independence and generalized white noise. We present a definition of stochastic independence which generalises the classical notion of stochastic independence and already anticipates the basic definition of quantum white noise.

Definition 3.1. Let $(\mathcal{A}, \varphi)$ be a probability space with three von Neumann subalgebras $\mathcal{A}_{i} \subseteq \mathcal{A}$ for which the conditional expectation $P_{i}:(\mathcal{A}, \varphi) \rightarrow \mathcal{A}_{i}$ exists $(i=0,1,2)$. The subalgebras $\mathcal{A}_{1}$ and $\mathcal{A}_{2}$ are independent over $\mathcal{A}_{0}$ if the following condition is satisfied:

$$
P_{0}(x y)=P_{0}(x) P_{0}(y) \quad \text { for any } x \in \mathcal{A}_{1}, y \in \mathcal{A}_{2} \text {. }
$$

If $\operatorname{dim}\left(\mathcal{A}_{0}\right)=1$, then $P_{0}(x)=\varphi(x) \cdot \mathbb{1}$ for all $x \in \mathcal{A}$ and we retain the notion of independence w.r.t. the state $\varphi$. If $(\mathcal{A}, \varphi)$ is a finite von Neumann algebra $\mathcal{A}$ with a faithful normal trace $\varphi$, the definition of independence rephrases the properties of a commuting square (cf. [GHJ]).

Now we are in the position to give the fundamental definition of quantum white noise. Its structural properties will enable us to develop a theory of stochastic integration as well as to construct stationary quantum Markov processes in the sense of [Kü1, Kü2].

Definition 3.2. A quantum or generalized white noise (over the von Neumann algebra $\left.\mathcal{A}_{0}\right)$ is a quadruple $\left(\mathcal{A}, \varphi, S_{t} ;\left(\mathcal{A}_{I}\right)_{I}\right)$, where $\left(\mathcal{A}, \varphi, S_{t}\right)$ is a stationary dynamical system and $\left(\mathcal{A}_{I}\right)_{I \subset \mathbb{R}}$ is a filtration of the probability space $(\mathcal{A}, \varphi)$ such that

(i) $S_{t} \circ P_{0}=P_{0}$, and $S_{t}\left(\mathcal{A}_{I}\right)=\mathcal{A}_{I+t}$ for $t \in \mathbb{R}$, where $I+t:=\{s+t \mid s \in I\}$,

(ii) $\mathcal{A}_{[r, s]}$ and $\mathcal{A}_{\left[r^{\prime}, s^{\prime}\right]}$ are independent over $\mathcal{A}_{0}$ in $(\mathcal{A}, \varphi)$, whenever $s \leq r^{\prime}$.

From now on we suppress the attribute 'quantum' and simply speak of a white noise (over $\mathcal{A}_{0}$ ) and a stationary Markov process. Of special interest for the construction of stationary Markov processes is the following object.

Definition 3.3. Let $\left(\mathcal{A}, \varphi, S_{t} ;\left(\mathcal{A}_{I}\right)_{I}\right)$ be a white noise. A unitary (left) cocycle $u$ (w.r.t. $S_{t}$ ) is a weak ${ }^{*}$-continuous family $\left(u_{t}\right)_{t \geq 0} \subset \mathcal{A}$ of unitaries with the following properties:

(i) $u_{t} \in \mathcal{A}_{[0, t]}$ for any $t \geq 0$ (adaptedness),

(ii) $u_{s+t}=S_{t}\left(u_{s}\right) u_{t}$ for any $s, t \geq 0$ (cocycle identity).

Putting $C_{t}:=\operatorname{Ad} u_{t}$ for $t \geq 0$ and $C_{t}:=S_{t} \circ C_{-t}^{-1} \circ S_{-t}$ for $t<0$, then $C \subset \operatorname{Aut}(\mathcal{A}, \varphi)$ iff $u \subset \mathcal{A}^{\varphi}$. If we agree on $T_{t}:=C_{t} \circ S_{t} \in \operatorname{Aut}(\mathcal{A}, \varphi)$, we get the following

Lemma 3.1. Let $\left(\mathcal{A}, \varphi, S_{t} ;\left(\mathcal{A}_{I}\right)_{I}\right)$ be a white noise over $\mathcal{A}_{0}$ and $u \subset \mathcal{A}^{\varphi}$ a unitary cocycle w.r.t. $S_{t}$. Then $\left(\mathcal{A}, \varphi, T_{t} ; \mathcal{A}_{0}\right)$ is a stationary Markov process with values in $\mathcal{A}_{0}$.

$\left(\mathcal{A}, \varphi, T_{t} ; \mathcal{A}_{0}\right)$ is also called a coupling to white noise $\left(\mathcal{A}, \varphi, S_{t} ;\left(\mathcal{A}_{I}\right)_{I}\right)$ and $S_{t}$ is called the free evolution for the stationary Markov process $\left(\mathcal{A}, \varphi, T_{t} ; \mathcal{A}_{0}\right)$. 
Finally, we mention that our definition of white noise is not a mere question of definition. It is the outcome of a structural identification of stationary Markov processes as couplings to white noise. For a large class of stationary Markov processes such a structure can be established. As an example we mention ([Kü2]):

Theorem 3.2. Let $\left(\mathcal{A}, \varphi, T_{t} ; M_{n}\right)$ be a stationary Markov process with values in the $n \times n$-matrices $M_{n}$. If there exists a projection $e \in M_{n}$ which is minimal in $M_{n}$ and invariant under $T_{t}$, then the process is a coupling to white noise.

This result features that the Markov property of the considered process is reflected by the white noise property of the free evolution and conversely. Thus the stochastic properties of a stationary process and of its free evolution are intimately related although the free evolution is not unique.

4. Stochastic integration on Hilbert modules. In the case of Gaussian white noise, stochastic integration of Itô type is deeply related to the notion of stochastic independence, which leads to a factorisation (w.r.t. the state) of the product of two independent random variables. In our setting of a quantum white noise $\left(\mathcal{A}, \varphi, S_{t},\left(\mathcal{A}_{I}\right)_{I}\right)$ over the von Neumann algebra $\mathcal{A}_{0}$, such a factorisation of independent elements w.r.t. $\varphi$, in general, is no longer present. Here we can only expect a factorisation of independent elements in $\mathcal{A}$ w.r.t. the conditional expectation $P_{0}:(\mathcal{A}, \varphi) \rightarrow \mathcal{A}_{0}$. This observation naturally leads to the concept of Hilbert module as substitute for the GNS Hilbert space of white noise if one wants to define stochastic integrals of Itô type.

4.1. Hilbert modules. We briefly introduce Hilbert modules to the extent necessary for our later purposes. For a more detailed approach to Hilbert modules we refer the reader to $[\mathrm{Fr}, \mathrm{La}, \mathrm{Pa}, \mathrm{Sc}]$ and the references therein. We mention that Hilbert modules meanwhile appear also in the context of quantum stochastic integration on Fock modules (cf. $[\mathrm{AcLu}, \mathrm{Sk}]$ ).

Let $(\mathcal{A}, \varphi)$ be a probability space and $\mathcal{A}_{0}$ a von Neumann subalgebra of $\mathcal{A}$ such that the conditional expectation $P_{0}:(\mathcal{A}, \varphi) \rightarrow \mathcal{A}_{0}$ exists. Denote by $p_{0} \in \mathcal{B}\left(L^{2}(\mathcal{A}, \varphi)\right)$ the projection induced by $P_{0}$. Given the right action of $\mathcal{A}_{0}$ on $\mathcal{A} p_{0}$ by $\mathcal{A} p_{0} \times \mathcal{A}_{0} \ni\left(x p_{0}, a_{0}\right) \mapsto$ $x p_{0} a_{0}=x a_{0} p_{0} \in \mathcal{A} p_{0}$ and the left action of $a \in \mathcal{A}$ on $x \in \mathcal{A} p_{0}$ by $a x$, we obtain an $\mathcal{A}$ - $\mathcal{A}_{0}$-bimodule $\mathcal{A} p_{0}$. We introduce the $\mathcal{A}_{0}$-valued inner product

$$
\langle x \mid y\rangle_{0}:=x^{*} y \quad \text { for any } x, y \in \mathcal{A} p_{0},
$$

where we identify $\mathcal{A}_{0} p_{0}$ with $\mathcal{A}_{0}$. Denote the closure of $\mathcal{A} p_{0}$ in the strong operator topology (s-topology) on $\mathcal{B}\left(L^{2}(\mathcal{A}, \varphi)\right)$ by

$$
L^{2}\left(\mathcal{A}, P_{0}\right):=\left\{\mathcal{A} p_{0}\right\}^{-s} .
$$

Carrying out the extension of the $\mathcal{A}_{0}$-valued inner product $\langle\cdot \mid \cdot\rangle_{0}$ on $\mathcal{A} p_{0}$ and of the right action of $\mathcal{A}_{0}$ on $\mathcal{A} p_{0}$ to $L^{2}\left(\mathcal{A}, P_{0}\right)$ and denoting them by the same symbols, $L^{2}\left(\mathcal{A}, P_{0}\right)$ is a (right) Hilbert $W^{*}$-module over $\mathcal{A}_{0}$, which we call for brevity $(G N S)$ Hilbert module. Furthermore we agree on $|x|_{0}:=\langle y \mid y\rangle_{0}^{1 / 2}$ and define on $L^{2}\left(\mathcal{A}, P_{0}\right)$ the norm $\|x\|_{0}:=\left\||x|_{0}\right\|$. We remark that the s-topology on $L^{2}\left(\mathcal{A}, P_{0}\right)$ is induced by the family of seminorms $d_{\xi}(x):=\left\||x|_{0} \xi\right\|, \xi \in L^{2}(\mathcal{A}, \varphi), x \in L^{2}\left(\mathcal{A}, P_{0}\right)$. In addition, because we restrict our- 
selves to von Neumann algebras with separable predual, elements of the Hilbert module $L^{2}\left(\mathcal{A}, P_{0}\right)$ can be approximated in the s-topology by $\|\cdot\|_{0}$-bounded sequences in $\mathcal{A}$.

Let $\mathcal{B}\left(L^{2}\left(\mathcal{A}, P_{0}\right)\right)$ be the set of adjointable $\mathcal{A}_{0}$-linear maps on the Hilbert module $L^{2}\left(\mathcal{A}, P_{0}\right)$ and denote the unique adjoint of an adjointable map $L$ by $L^{*}$. Then the left action of $a \in \mathcal{A}$ on $\mathcal{A} p_{0}$ lifts to an element $L_{a} \in \mathcal{B}\left(L^{2}\left(\mathcal{A}, P_{0}\right)\right)$ such that

$$
\left\langle y \mid L_{a}(x)\right\rangle_{0}=\left\langle L_{a}^{*}(y) \mid x\right\rangle_{0}=\left\langle L_{a^{*}}(y) \mid x\right\rangle_{0} \quad \text { for any } x, y \in L^{2}\left(\mathcal{A}, P_{0}\right) .
$$

From now on we simply write $L_{a}(x)$ as ax. In addition, a morphism $T$ of $(\mathcal{A}, \varphi)$, which commutes with the modular automorphism group of $(\mathcal{A}, \varphi)$ and leaves $\mathcal{A}_{0}$ pointwise fixed, extends uniquely to an adjointable map on $L^{2}\left(\mathcal{A}, P_{0}\right)$ which we still denote by $T$.

4.2. Quantum white noise and Hilbert modules. Let $\left(\mathcal{A}, \varphi, S_{t} ;\left(\mathcal{A}_{I}\right)_{I}\right)$ be a white noise over $\mathcal{A}_{0}$. The conditional expectations $P_{I}:(\mathcal{A}, \varphi) \rightarrow \mathcal{A}_{I}$ and the group of automorphisms $S_{t}$ commute with the modular automorphism group of $(\mathcal{A}, \varphi)$ and leave $\mathcal{A}_{0}$ pointwise fixed. Hence, they extend to adjointable maps $P_{I}$ and $S_{t}$ on $L^{2}\left(\mathcal{A}, P_{0}\right)$. In particular the filtration $\left(\mathcal{A}_{I}\right)_{I}$ extends to a filtration $\left(L^{2}\left(\mathcal{A}_{I}, P_{0}\right)\right)_{I}$ of $L^{2}\left(\mathcal{A}, P_{0}\right)$ which is given by the Hilbert modules $L^{2}\left(\mathcal{A}_{I}, P_{0}\right):=P_{I}\left(L^{2}\left(\mathcal{A}, P_{0}\right)\right)=\left\{\mathcal{A}_{I} p_{0}\right\}^{-s}$. Here, we identify $L^{2}\left(\mathcal{A}_{0}, P_{0}\right)$ with $\mathcal{A}_{0}$. We call two elements $x, y \in L^{2}\left(\mathcal{A}, P_{0}\right)$ independent if $x \in L^{2}\left(\mathcal{A}_{I}, P_{0}\right)$ and $y \in L^{2}\left(\mathcal{A}_{J}, P_{0}\right)$, where $I$ and $J$ are intervals which are disjoint or have one point in common (in these cases we write $|I \cap J|=0$ ).

Lemma 4.1. If $x \in L^{2}\left(\mathcal{A}_{I}, P_{0}\right)$ and $y \in L^{2}\left(\mathcal{A}_{J}, P_{0}\right)$ with $|I \cap J|=0$, then

$$
\langle x \mid y\rangle_{0}=\langle x \mid \mathbb{1}\rangle_{0}\langle\mathbb{1} \mid y\rangle_{0} .
$$

In addition to the factorisation property of independent elements in the Hilbert module $L^{2}\left(\mathcal{A}_{0}, P_{0}\right)$ we formulate a product of independent elements.

Lemma 4.2. Let $x \in L^{2}\left(\mathcal{A}_{I}, P_{0}\right)$ and $y \in L^{2}\left(\mathcal{A}_{J}, P_{0}\right)$ be independent, let $\left(x_{n}\right)_{n \in \mathbb{N}} \subset$ $\mathcal{A}_{I}$ be a sequence such that $x=s-\lim _{n \rightarrow \infty} x_{n}$. Then

$$
x \cdot y:=s-\lim _{n \rightarrow \infty} x_{n} y
$$

defines a product of independent elements in $L^{2}\left(\mathcal{A}, P_{0}\right)$ which does not depend on the chosen approximation $\left(x_{n}\right)_{n \in \mathbb{N}}$ and satisfies the equation

$$
|x \cdot y|_{0}^{2}=\left\langle\left. y|| x\right|_{0} ^{2} y\right\rangle_{0} .
$$

4.3. Stochastic integration on $L^{2}\left(\mathcal{A}, P_{0}\right)$. In the Hilbert module $L^{2}\left(\mathcal{A}, P_{0}\right)$ we find the (noncommutative) counterpart to Brownian motion as increment process of stochastic Itô integrals on the GNS Hilbert space of Gaussian white noise.

Definition 4.1. An additive cocycle $b$ with respect to the white noise $\left(\mathcal{A}, \varphi, S_{t}\right.$; $\left.\left(\mathcal{A}_{I}\right)_{I}\right)$ is a family $\left(b_{t}\right)_{t \geq 0} \subseteq L^{2}\left(\mathcal{A}, P_{0}\right)$ with the following properties

(i) $b_{t} \in L^{2}\left(\mathcal{A}_{[0, t]}, P_{0}\right)$ for any $t \geq 0$ (adaptedness),

(ii) $b_{s+t}=b_{t}+S_{t}\left(b_{s}\right)$ for any $s, t \geq 0$ (cocycle identity).

If in addition, $P_{0}\left(b_{t}\right)=0$ for any $t \geq 0$, then $b$ is called centred. 
We remark that centred additive cocycles $b$ enjoy the martingale property: $P_{[0, t]}\left(b_{t+s}\right)$ $=b_{t}$. Moreover, the pointwise continuity of $t \mapsto P_{[0, t]}$ in the s-topology on the Hilbert module $L^{2}\left(\mathcal{A}, P_{0}\right)$ yields $\left\langle b_{t} \mid a_{0} b_{t}\right\rangle_{0}=t \cdot\left\langle b_{1} \mid a_{0} b_{1}\right\rangle_{0}=: t \cdot \Lambda\left(a_{0}\right), a_{0} \in \mathcal{A}_{0}$.

Now we are prepared to formulate in $L^{2}\left(\mathcal{A}, P_{0}\right)$ left and right integrals w.r.t. centred additive cocycles $b$

$$
\int_{0}^{t} d b_{s} \cdot x_{s}, \quad \int_{0}^{t} x_{s} \cdot d b_{s}
$$

Here $\left(x_{s}\right)_{s \geq 0} \subseteq L^{2}\left(\mathcal{A}, P_{0}\right)$ means an adapted stochastic process, i.e. $x_{s} \in L^{2}\left(\mathcal{A}_{[0, s]}, P_{0}\right)$, which is required to be continuous in the s-topology or even in the $\|\cdot\|_{0}$-topology on $L^{2}\left(\mathcal{A}, P_{0}\right)$. (The integrals can be extended to larger classes of processes but in the present context we make no use of this possibility.) As usual, one first defines integrals for simple adapted processes $x$ with $x_{s}=\sum_{i=1}^{n} x_{s_{i}} \chi_{\left[s_{i-1}, s_{i}\right)}$, where $x_{s_{i}} \in L^{2}\left(\mathcal{A}_{\left[0, s_{i}\right]}, P_{0}\right)$. By means of the product of $x_{s_{i}}$ and $S_{s_{i}}\left(b_{s_{i+1}-s_{i}}\right)$ in $L^{2}\left(\mathcal{A}, P_{0}\right)$ simple stochastic integrals are well defined:

$$
\int_{0}^{t} d b_{s} \cdot x_{s}:=\sum_{i=1}^{n} S_{s_{i}}\left(b_{s_{i+1}-s_{i}}\right) \cdot x_{s_{i}}, \quad \int_{0}^{t} x_{s} \cdot d b_{s}:=\sum_{i=1}^{n} x_{s_{i}} \cdot S_{s_{i}}\left(b_{s_{i+1}-s_{i}}\right) .
$$

We obtain the following Itô isometries.

LEMMA 4.3. The simple left and right integrals w.r.t. a centred additive cocycle $b$ enjoy the $\mathcal{A}_{0}$-valued isometry identities

$$
\left|\int_{0}^{t} d b_{s} \cdot x_{s}\right|_{0}^{2}=\int_{0}^{t}\left|\Lambda(\mathbb{1})^{1 / 2} x_{s}\right|_{0}^{2} d s, \quad\left|\int_{0}^{t} x_{s} \cdot d b_{s}\right|_{0}^{2}=\int_{0}^{t} \Lambda\left(\left|x_{s}\right|_{0}^{2}\right) d s .
$$

These isometries are decisive for extending the integrals from simple stochastic processes to larger classes. We omit details and briefly state: Adapted processes which are continuous in the s-topology are approximated by simple processes $\left(x_{s}^{n}\right)_{s \geq 0}$ in the topology induced by the family of seminorms $\int_{0}^{t} d_{\xi}\left(x_{s}\right)^{2} d s$. In addition, we note that the definition of stochastic integrals extends straightforward to noncentred additive cocycles $\beta$.

4.4. Stochastic differential equations. Having established stochastic left and right integrals on $L^{2}\left(\mathcal{A}, P_{0}\right)$ one defines stochastic differential equations. We restrict our investigations to equations of the following type:

$$
x_{t}=x_{t_{0}}+\int_{t_{0}}^{t} d \beta_{s} \cdot a\left(s, x_{s}\right) .
$$

The function $a: \mathbb{R}^{+} \times L^{2}\left(\mathcal{A}, P_{0}\right) \rightarrow L^{2}\left(\mathcal{A}, P_{0}\right)$ respects adaptedness: for each $t \geq 0$ and each $x \in L^{2}\left(\mathcal{A}_{[0, t]}, P_{0}\right)$ again $a(t, x)$ is in $L^{2}\left(\mathcal{A}_{[0, t]}, P_{0}\right)$. Now we are capable to answer the question whether a solution of a stochastic differential equation exists and whether it is unique.

TheOREM 4.4. Let $a: \mathbb{R}^{+} \times L^{2}\left(\mathcal{A}, P_{0}\right) \rightarrow L^{2}\left(\mathcal{A}, P_{0}\right)$ be an adapted function with the following properties: For each interval $\left[t_{0}, t_{1}\right], t_{0} \geq 0$, there exists a number $C>0$ and for each $\xi \in L^{2}\left(\mathcal{A}_{0}, \varphi\right)$ there exists a continuous function $f_{\xi}$ on $\mathbb{R}^{+}$, such that for any 
$x, y \in L^{2}\left(\mathcal{A}, P_{0}\right), s, t \in\left[t_{0}, t_{1}\right]$, the following estimates hold:

$$
\begin{aligned}
d_{\xi}(a(t, x)-a(s, x)) & \leq C \cdot\left(1+d_{\xi}(x)\right) \cdot\left|f_{\xi}(t)-f_{\xi}(s)\right|, \\
\|a(t, x)-a(t, y)\|_{0} & \leq C \cdot\|x-y\|_{0} .
\end{aligned}
$$

If all these conditions are satisfied, the stochastic differential equation (4.1) has one and only one solution $x \subset L^{2}\left(\mathcal{A}, P_{0}\right)$; and this solution is $\|\cdot\|_{0}$-continuous.

4.5. Additive cocycles and their construction. We have seen that in the GNS Hilbert module $L^{2}\left(\mathcal{A}, P_{0}\right)$ of the white noise $\left(\mathcal{A}, \varphi, S_{t},\left(\mathcal{A}_{I}\right)_{I}\right)$ and in the presence of a centred additive cocycle, a theory of stochastic integration can be established. The question arises: Where does an additive cocycle come from? Indeed, we are now going to show that from a unitary cocycle in the Hilbert module an additive cocycle can be constructed.

Definition 4.2. An isometric cocycle $u$ is a family $\left(u_{t}\right)_{t \geq 0} \subseteq L^{2}\left(\mathcal{A}, P_{0}\right)$ such that for any $s, t \geq 0$

(i) $\left|u_{t}\right|_{0}=\mathbb{1}$,

(ii) $u_{t} \in L^{2}\left(\mathcal{A}_{[0, t]}, P_{0}\right)$,

(iii) $u_{s+t}=S_{t}\left(u_{s}\right) \cdot u_{t}$.

Of course, a unitary cocycle $u \subseteq \mathcal{A}$ is an isometric one.

THEOREM 4.5. There is a one-to-one correspondence between $\|\cdot\|_{0}$-continuous isometric cocycles $u \subset L^{2}\left(\mathcal{A}, P_{0}\right)$ and additive cocycles $\beta_{t}=b_{t}+K t$, consisting of a centred additive cocycle $b \subset L^{2}\left(\mathcal{A}, P_{0}\right)$ and an operator $K \in \mathcal{A}_{0}$ with $-\left(K+K^{*}\right)=\left|b_{1}\right|_{0}^{2}$. The cocycle $\beta$ associated with $u$ is given by

$$
\beta_{t}=\|\cdot\|_{0}-\lim _{n \rightarrow \infty} \sum_{j=0}^{n-1} S_{j t / n}\left(u_{t / n}-\mathbb{1}\right)
$$

and $K$ satisfies $P_{0}\left(u_{t}\right)=\exp (K t) \in \mathcal{A}_{0}$. Conversely, if $\beta$ is given, the corresponding $u$ solves the stochastic differential equation

$$
u_{t}=\mathbb{1}+\int_{0}^{t} d \beta_{s} \cdot u_{s} .
$$

Moreover, $u$ defines the $\|\cdot\|$-continuous $W^{*}$-dynamical semigroup $R_{t}(a):=\left\langle u_{t} \mid a u_{t}\right\rangle_{0}$ on $\mathcal{A}_{0}$ with generator $L(a):=\Lambda(a)+K^{*} a+a K$, where $\Lambda(a):=\left\langle b_{1} \mid a b_{1}\right\rangle_{0}$.

5. Additive cocycles and their mutual quadratic variation. By virtue of theorem 4.5 a bijective correspondence already holds between additive and isometric cocycles in the Hilbert module $L^{2}\left(\mathcal{A}, P_{0}\right)$ of a white noise $\left(\mathcal{A}, \varphi, S_{t},\left(\mathcal{A}_{I}\right)_{I}\right)$. If we want to ensure that the solution of the stochastic differential equation (4.2) yields a unitary cocycle $u$, the corresponding additive cocycle $\beta$ has to be subjected to further conditions. To formulate these conditions, it is necessary to take the product of two elements of $L^{2}\left(\mathcal{A}, P_{0}\right)$ which are not independent. In the case of Gaussian white noise, Brownian motion $B$ is not only contained in $L^{2}\left(\mathcal{S}^{\prime}, \Sigma, \mu\right)$, but even in $L^{4}\left(\mathcal{S}^{\prime}, \Sigma, \mu\right)$ (cf. [Hi]). Consequently, in our general context of a white noise $\left(\mathcal{A}, \varphi, S_{t} ;\left(\mathcal{A}_{I}\right)_{I}\right)$, we look for a space $\mathcal{E}^{4} \subset L^{2}\left(\mathcal{A}, P_{0}\right)$ which plays the role of $L^{4}\left(\mathcal{S}^{\prime}, \Sigma, \mu\right)$ in the case of Gaussian white noise. Since we are 
interested in the construction of stationary Markov processes, it is no loss of generality to assume that $\mathcal{A}$ is a finite von Neumann algebra and $\varphi$ acts as a tracial state on $\mathcal{A}$. For brevity, a densely defined, closed operator $X$ which is affiliated to $\mathcal{A}$ and contains $L^{2}\left(\mathcal{A}_{0}, \varphi\right)$ in its core will be called $P_{0}$-affiliated.

Proposition 5.1. Let $(\mathcal{A}, \varphi)$ be a von Neumann algebra $\mathcal{A}$ equipped with a faithful normal trace $\varphi$ and denote by $\mathcal{A}_{0}$ a von Neumann subalgebra of $\mathcal{A}$.

(i) There is a one-to-one correspondence between $x \in L^{2}\left(\mathcal{A}, P_{0}\right)$ and $P_{0}$-affiliated operators $X$, which is given by $X a^{\prime} \xi_{0}=a^{\prime} x \xi_{0}$, for any $a^{\prime} \in \mathcal{A}^{\prime}, \xi_{0} \in L^{2}\left(\mathcal{A}_{0}, \varphi\right)$.

(ii) $\mathcal{E}^{4}:=\left\{x \in L^{2}\left(\mathcal{A}, P_{0}\right) \mid x=s-\lim _{n \rightarrow \infty} x_{n}, \sup _{n \in \mathbb{N}}\left\{\left\|x_{n}^{*} x_{n}\right\|_{0},\left\|x_{n} x_{n}^{*}\right\|_{0}\right\}<\infty, x_{n} \in\right.$ $\mathcal{A}\}$ enjoys the following properties:

- To each $x \in \mathcal{E}^{4}$ with $X$ as the associated $P_{0}$-affiliated operator, there uniquely exists $x^{*} \in \mathcal{E}^{4}$ such that its corresponding $P_{0}$-affiliated operator is given by $X^{*}$. Moreover, if $x=s-\lim _{n \rightarrow \infty} x_{n}$ then $x^{*}=s-\lim _{n \rightarrow \infty} x_{n}^{*}$, and the limits are independent of the chosen approximation $\left(x_{n}\right)_{n \in \mathbb{N}} \subset \mathcal{A}$.

- For any two elements $x, y \in \mathcal{E}^{4}$ there exists a well defined product $x y \in$ $L^{2}(\mathcal{A}, \varphi)$; the corresponding affiliated operator with $\Omega$ in its domain is given by $X Y$, the product of the operators $X$ and $Y$ belonging to $x$ and $y$.

Due to this correspondence between elements in $L^{2}\left(\mathcal{A}, P_{0}\right)$, respectively in $L^{2}(\mathcal{A}, \varphi)$, and affiliated operators we will no longer distinguish between these two objects. Now we study the properties of additive cocycles in $\mathcal{E}^{4}$. As mentioned above we can restrict our considerations to a white noise $\left(\mathcal{A}, \varphi, S_{t},\left(\mathcal{A}_{I}\right)_{I}\right)$ with tracial state $\varphi$.

TheOREM 5.2. Let $\beta$ and $\gamma$ be additive cocycles in $\mathcal{E}^{4} \subset L^{2}\left(\mathcal{A}, P_{0}\right)$. Then

(i) the mutual quadratic variation $[\beta, \gamma]$ of the additive cocycles $\beta$ and $\gamma$ exists in $L^{2}(\mathcal{A}, \varphi)$ and is given by

$$
[\beta, \gamma]_{t}:=\lim _{|\mathcal{Z}| \rightarrow 0} \sum_{i<n_{\mathcal{Z}}}\left(\beta_{t_{i+1}}-\beta_{t_{i}}\right)\left(\gamma_{t_{i+1}}-\gamma_{t_{i}}\right)
$$

where $\mathcal{Z}:=\left\{t_{i} \mid 0=t_{0}<t_{1}<\ldots<t_{n_{\mathcal{Z}}}=t\right\}$ and $|\mathcal{Z}|$ denotes the mesh of the partition $\mathcal{Z}$

(ii) the equation $\beta_{t} \gamma_{t}=[\beta, \gamma]_{t}+\int_{0}^{t} d \beta_{s} \cdot \gamma_{s}+\int_{0}^{t} \beta_{s} \cdot d \gamma_{s}$ holds and

(iii) if $\beta$ and $\gamma$ are independent, then $[\beta, \gamma]=0$.

If $\left(\beta^{i}\right)_{i=1, \ldots, n}$ are additive cocycles in $\mathcal{E}^{4}$, then $\left(\left[\beta^{i}, \beta^{j}\right]\right)_{i, j=1, \ldots, n}$ is a family of additive cocycles in $L^{2}(\mathcal{A}, \varphi)$ which forms an (abstract) Itô table (cf. [AFQ]).

If $\mathcal{A}_{0}$ is finite dimensional and the mutual quadratic variation $[\beta, \gamma]$ of two additive cocycles $\beta$ and $\gamma$ is a linear combination of $\beta, \gamma, \beta^{*}, \gamma^{*}$ and cocycles $K \cdot t, K \in \mathcal{A}_{0}$, then $\left\{\beta, \gamma, \mathcal{A}_{0}\right\}$ forms a ${ }^{*}$-algebra with respect to the mutual quadratic variation as multiplication. The generalization for more than two additive cocycles is obvious. In the context of Fock space stochastic integration this kind of *-algebra is known as Itô algebra (cf. [Be]).

6. The main result. We now formulate a bijective correspondence between additive and multiplicative cocycles with respect to the free evolution of a white noise. It is, in 
particular, designed for the construction of stationary Markov processes. Note that in the following theorem $\mathcal{E}^{4}$ is defined w.r.t. the centralizer $\mathcal{A}^{\varphi}$ of $(\mathcal{A}, \varphi)$.

Theorem 6.1. Let $\left(\mathcal{A}, \varphi, S_{t} ;\left(\mathcal{A}_{I}\right)_{I}\right)$ be a white noise over $\mathcal{A}_{0}$. There is a canonical bijective correspondence between

(i) $\|\cdot\|_{0}$-continuous unitary cocycles $u \subseteq \mathcal{A}^{\varphi}$ and

(ii) additive cocycles $\beta \subseteq \mathcal{E}^{4}$ with $\left[\beta^{*}, \beta\right]_{t}=-\left(\beta_{t}^{*}+\beta_{t}\right)$.

The correspondence is given as follows: $u$ is the solution of the stochastic differential equation

$$
u_{t}=\mathbb{1}+\int_{0}^{t} d \beta_{s} \cdot u_{s}
$$

and $\beta$ is constructed from $u$ by

$$
\beta_{t}=\|\cdot\|_{0}-\lim _{n \rightarrow \infty} \sum_{j=0}^{n-1} S_{j t / n}\left(u_{t / n}-\mathbb{1}\right) .
$$

For practical purposes it is not necessary to calculate the mutual quadratic variation if one wants to verify condition (ii) of the preceding theorem.

Corollary 6.2. Defining $K:=P_{0}\left(\beta_{1}\right), b_{t}:=\beta_{t}-K t \in \mathcal{E}^{4}$ and $F(t):=\left\|b_{t}^{*} b_{t} \Omega\right\|^{2}$, condition (ii) of Theorem 6.1 is equivalent to

(i) $\left|b_{t}\right|_{0}^{2}=-\left(K^{*}+K\right) \cdot t$

(ii) $\left\|\left(b_{t}^{*}+b_{t}\right) \Omega\right\|^{2}=F^{\prime}(0) \cdot t$,

(iii) $\left\|\left(b_{t}^{*}+b_{t}\right) \Omega\right\|^{2}=-\left\langle b_{t}^{*} b_{t} \Omega \mid\left(b_{t}^{*}+b_{t}\right) \Omega\right\rangle$.

Corollary 6.2 delivers convenient criteria for additive cocycles such that the solution of equation (6.3) is a unitary cocycle. These conditions only require knowledge about the centralizer of a white noise and the existence of the fourth moment $F(t)$ of the additive cocycle $b$.

COROLlary 6.3. (i) If either one of the conditions in Theorem 6.1 is fulfilled, then $\left(\mathcal{A}, \varphi, T_{t} ; \mathcal{A}_{0}\right)$, where $T_{t}:=\mathrm{Ad} u_{t} \circ S_{t}$, is a stationary Markov process with values in $\mathcal{A}_{0}$. The generator $L$ of the semigroup $R_{t}:=P_{0} \circ T_{t} \circ P_{0}$ on $\left(\mathcal{A}_{0}, \varphi\right)$ is given by

$$
L(a):=\left\langle b_{1} \mid a b_{1}\right\rangle_{0}+K^{*} a+a K .
$$

(ii) If $\beta^{i}, i=1, \ldots, n$, are mutual independent additive cocycles, all satisfying condition (ii) of Theorem 6.1, then so does $\beta:=\sum_{i=1}^{n} \beta^{i}$. In this case the generator of the associated semigroup is the sum of the generators associated with $\beta^{i}(i=1, \ldots, n)$.

The composition of independent additive cocycles $\beta^{i}$ easily extends to $\|\cdot\|_{0}$-bounded sums.

COROllary 6.4. Let the cocycle $\beta_{t}=b_{t}+$ Kt satisfy condition (ii) of Theorem 6.1. Then $b_{t}^{*}=-b_{t}$ implies that the associated $W^{*}$-dynamical semigroup $e^{t L}$ obeys detailed balance in the sense of Kossakowski et al. ([KFGV]). 
7. Examples. In the cases of squeezed white noise and q-white noise we finally illustrate the handling of the presented general theory. We construct simple examples for generators of semigroups $R_{t}=e^{t L}$ on $\left(M_{n}, \rho\right)$, which come from stationary Markov processes.

7.1. Squeezed white noise. Let $(\mathcal{C}, \psi)$ be the GNS representation of the CCR algebra over $L^{2}(\mathbb{R})$ w.r.t. the faithful quasifree state $\psi$ defined on the Weyl operators $W(f)$, $f \in L^{2}(\mathbb{R})$, by

$$
\psi(W(f)):=\exp \left[-\frac{\lambda}{4}\left(\cosh (2 r)\|f\|^{2}+\sinh (2 r) \operatorname{Re}\left(e^{i \theta}\langle f \mid J f\rangle\right)\right)\right] .
$$

Here $J$ is the canonical conjugation on $L^{2}(\mathbb{R})$ and $r, \theta \in \mathbb{R}, \lambda>1$. The spectrum of the modular group w.r.t. $\psi$ is given by $\left\{\left(\frac{\lambda+1}{\lambda-1}\right)^{i n t} \mid n \in \mathbb{Z}\right\}$. A filtration $\left(\mathcal{C}_{I}\right)_{I}$ on $(\mathcal{C}, \psi)$ is induced by $\left(L^{2}(I)\right)_{I}$ in the usual way. Finally, let $S_{t}$ be the second quantisation of the shift on $L^{2}(\mathbb{R})$. Then $\left(M_{n} \otimes \mathcal{C}, \rho \otimes \psi, i d \otimes S_{t} ;\left(M_{n} \otimes \mathcal{C}_{I}\right)_{I}\right)$ is a white noise over $M_{n}$, which is an amplification of squeezed white noise $\left(\mathcal{C}, \varphi, S_{t},\left(\mathcal{C}_{I}\right)_{I}\right)$ (cf. [Ba], [HKR]). Let $l_{1}$ and $l_{2}$ be elements of the eigenspaces of $\sigma_{t}^{\rho}$ with the eigenvalues $\kappa^{i t}$ and $\kappa^{-i t}$. Choose $\lambda>1$ such that $\kappa=\frac{\lambda+1}{\lambda-1}$, then

$$
b_{t}:=l_{1} \otimes A_{\psi}\left(\chi_{[0, t]}\right)+l_{2} \otimes A_{\psi}^{*}\left(\chi_{[0, t]}\right)
$$

is a centred additive cocycle in $\mathcal{E}^{4}$ (here $A_{\psi}, A_{\psi}^{*}$ denote annihilation and creation operator in the GNS representation of $(\mathcal{C}, \psi)$ ). The fourth moment $F(t)$ of $b_{t}$ is of order $t^{2}$, hence $F^{\prime}(0)=0$. Applying Corollary 6.2 yields $b_{t}^{*}=-b_{t}$, consequently $l:=-l_{2}=l_{1}^{*}$. Condition (i) determines $K$ up to a skew selfadjoint operator $i H$. This leads to the generator

$$
L(x):=i[H, x]+R^{*} x R+\kappa^{-1 / 2} R x R^{*}-\frac{1}{2}\left\{R^{*} R, x\right\}-\frac{1}{2} \kappa^{-1 / 2}\left\{R R^{*}, x\right\},
$$

where $R:=\sqrt{\frac{\lambda+1}{2}}\left(\cosh (r) \cdot l+\sinh (r) \cdot l^{*}\right)$ and $x \in M_{n}$. We remark that this construction parallels the one given in $[\mathrm{ApFr}]$ for Fermionic quasifree white noise.

7.2. q-white noise. We follow the presentation in $[\mathrm{BKS}]$. Let $\mathcal{H}:=L_{\mathbb{R}}^{2}(\mathbb{R}) \otimes \mathbb{R}^{n}$ and denote its complexification by $\mathcal{H}_{\mathbb{C}}$. The family of operators $\left\{a(f): f \in \mathcal{H}_{\mathbb{C}}\right\}$, which satisfy the $q$-commutation relation

$$
a(f) a^{*}(g)-q a^{*}(g) a(f)=\langle f \mid g\rangle \mathbb{1}, \quad-1<q<1,
$$

are realized as bounded operators on the $q$-Fock space $\mathcal{F}_{q}\left(\mathcal{H}_{\mathbb{C}}\right)$. Furthermore, $\Gamma_{q}(\mathcal{H}):=$ $\bigvee_{f \in \mathcal{H}}\left\{a(f)+a^{*}(f)\right\} \subset \mathcal{B}\left(\mathcal{F}_{q}\left(\mathcal{H}_{\mathbb{C}}\right)\right)$ is a finite von Neumann algebra and $\psi:=\langle\Omega \mid \cdot \Omega\rangle$ acts as a tracial state on $\Gamma_{q}(\mathcal{H})(\Omega$ is the vacuum vector $)$. The shift on $L^{2}(\mathbb{R})$ induces the shift $S_{t}$ on $\Gamma_{q}(\mathcal{H})$. Let $\rho$ be the trace on $M_{n}$. Then $\left(M_{n} \otimes \Gamma_{q}(\mathcal{H}), \rho \otimes \psi\right.$, id $\otimes S_{t},\left(M_{n} \otimes\right.$ $\left.\left.\Gamma_{q}\left(L^{2}(I) \otimes \mathbb{R}^{n}\right)\right)_{I}\right)$ is a white noise over $M_{n}$. With the canonical base $\left(e_{j}\right)_{j=1, \ldots, n}$ of $\mathbb{R}^{n}$ and $\left(l_{j}\right)_{j=1, \ldots, n} \subset M_{n}$,

$$
b_{t}^{j}:=i l_{j} \otimes\left(a\left(\chi_{[0, t]} \otimes e_{j}\right)+a^{*}\left(\chi_{[0, t]} \otimes e_{j}\right)\right), \quad j=1, \ldots, n,
$$

are mutual independent centred additive cocycles in $M_{n} \otimes \Gamma_{q}(\mathcal{H})$. The fourth moments of $b_{t}^{j}$ are easily computed to be of order $t^{2}$, hence $F^{\prime}(0)=0$. Applying corollary 6.2 immediately yields $l_{j}=l_{j}^{*}$. With some $H=H^{*} \in M_{n}$ this leads to generators of the form

$$
L(x):=i[H, x]+\sum_{j} l_{j} x l_{j}-\frac{1}{2}\left\{l_{j}^{2}, x\right\}, \quad \text { for any } x \in M_{n} .
$$




\section{References}

[AcLu] L. Accardi and Y.G. Lu, The Wigner semi-circle law in quantum electro dynamics, Comm. Math. Phys. 180, 605-632, 1996.

[AFL] L. Accardi, A. Frigerio and J. T. Lewis, Quantum stochastic processes, Publ. RIMS Kyoto 18, 97-133, 1982.

[AFQ] L. Accardi, F. Fagnola and J. Quaegebeur, A representation free quantum stochastic calculus, J. Funct. Anal. 104, 149-197, 1992.

[ApFr] D. Applebaum and A. Frigerio, Stationary dilations of $W^{*}$-dynamical systems constructed via quantum stochastic differential equations, in: From local times to global geometry, control and physics (Coventry, 1984/85), Pitman Res. Notes Math. Ser., 150, 1-38, Longman Sci. Tech., Harlow 1986.

[Ba] A. Barchielli, Applications of quantum stochastic calculus to quantum optics, Quantum probability \& related topics, QP-PQ, VI, 111-125, 1991.

[Be] V. P. Belavkin, A new form and a *-algebraic structure of integrals in Fock space, Rend. Sem. Mat. Fis. Milano 58, 177-193, 1988.

[BiSp] P. Biane and R. Speicher, Stochastic calculus with respect to free Brownian motion and analysis on Wigner space, preprint, Paris, 1997.

[BKS] M. Bożejko, B. Kümmerer and R. Speicher, q-Gaussian processes: non-commutative and classical aspects, Comm. Math. Phys. 185, 129-154, 1997.

[BoSp] M. Bożejko and R. Speicher, An example of generalized Brownian motion, Comm. Math. Phys. 137, 519-531, 1991.

[BSW] C. Barnett, R. F. Streater and I. F. Wilde, Quasi-free quantum stochastic integrals for the CAR and CCR, J. Funct. Anal. 52, 19-47, 1983.

[Fr] M. Frank, Self-duality and $C^{*}$-reflexivity of Hilbert $C^{*}$-moduli, Zeitschr. Anal. Anw. 9, 165-176, 1990.

[GHJ] F. M. Goodman, P. de la Harpe and V. F. R. Jones, Coxeter Graphs and Towers of Algebras, Springer Verlag, New York, 1989.

[HKR] J. Hellmich, R. Honegger, C. Köstler, B. Kümmerer, A. Rieckers and C. Rupp, The quantum stochastic calculus of classical and non-classical squeezed white noise, preprint, Tübingen, 1997.

[Hi] T. Hida, Brownian Motion, Springer Verlag, New York, 1980.

[HuLi] R. L. Hudson and J. M. Lindsay, A noncommutative martingale representation theorem for non-Fock quantum Brownian motion, J. Funct. Anal. 61, 202-221, 1985.

[KFGV] A. Kossakowski, A. Frigerio, V. Gorini and M. Verri, Quantum detailed balance and KMS condition, Comm. Math. Phys. 57, 97-110, 1977.

[Kü1] B. Kümmerer, Markov dilations on $W^{*}$-algebras, J. Funct. Anal. 63, 139-177, 1985.

[Kü2] B. Kümmerer, Stochastic processes with values in $M_{n}$ as couplings to free evolutions, preprint, 1993.

[KüMa] B. Kümmerer and H. Maassen, Elements of quantum probability, to appear in Quantum Probability Communications, X.

[KüSp] B. Kümmerer and R. Speicher, Stochastic integration on the Cuntz algebra $O_{\infty}$, J. Funct. Anal. 103, 372-408, 1992.

[La] E. C. Lance, Hilbert $C^{*}$-modules, London Mathematical Society Lecture Notes Series 210, 1995.

[Me] P. A. Meyer, Quantum Probability for Probabilists, Springer Verlag, Berlin, Heidelberg, 1993. 
[P] K. R. Parthasarathy, An Introduction to Quantum Stochastic Calculus, Birkhäuser, Basel, 1992.

[Pa] W. L. Paschke, Inner product modules over $B^{*}$-algebras, Trans. Amer. Math. Soc. 182, 443-468, 1973.

[Pr] J. Prin, Verallgemeinertes weißes Rauschen und nichtkommutative stochastische Integration, Diplomarbeit, Tübingen, 1989.

[Sc] J. Schweizer, Interplay between noncommutative topology and operators on $C^{*}$ algebras, thesis, Tübingen, 1996.

[Sk] M. Skeide, Hilbert modules in quantum electro dynamics and quantum probability, to appear in Comm. Math. Phys. 